\title{
Neue Tarifversion TARMED per 1.6.2012 - wichtige Änderungen
}

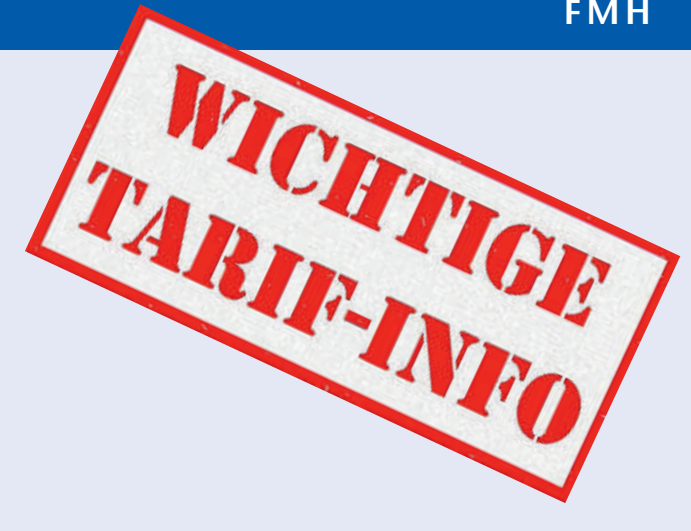

\section{Ernst Gähler ${ }^{a}$,}

Thomas Kessler $^{b}$

a Dr. med., Vizepräsident FMH, Verantwortlicher Ressort Tarife und Verträge

b Ressort Tarife und Verträge
Korrespondenz: Tarifdienst FMH Froburgstrasse 15 CH-4600 Olten Tel. 0622879696 Fax 0622879690 tarife[at]fmh.ch

\section{Kapitel 29 Schmerztherapie - Kapitelrevision}

Das ganze Kapitel 29 Schmerztherapie wurde umfassend überarbeitet. Neu umfasst das Hauptkapitel 29 Schmerztherapie zwei Unterkapitel: 29.05 Allgemeine Schmerztherapie und 29.06 Interventionelle Schmerzdiagnostik und -therapie.

Das Unterkapitel 29.05 beinhaltet im Speziellen die Allgemeine Schmerztherapie am Schädel (Unterkapitel 29.05.01), an der Wirbelsäule (Unterkapitel 29.05.02), Stamm, Schultergürtel, Beckengürtel und Extremitäten (Unterkapitel 29.05.03). Alle Tarifpositionen dieses Unterkapitels sind mit der qualitativen Dignität «Alle» versehen und können durch alle Ärzte erbracht und dem Kostenträger in Rechnung gestellt werden.

Das Unterkapitel 29.06 Interventionelle Schmerzdiagnostik und -therapie beinhaltet im Speziellen die Grundleistung (Unterkapitel 29.06.01), die interventionelle Schmerzdiagnostik und -therapie an der Wirbelsäule (Unterkapitel 29.06.02), an den sympathischen Ganglien, N. trigeminus, peripheren Nerven (Unterkapitel 29.06.03), die interventionelle Schmerztherapie unter Ultraschallkontrolle (Unterkapitel 29.06.04) und die intravenöse Regionalanalgesie (Unterkapitel 29.06.05). Alle Tarifpositionen dieses Unterkapitels sind mit der qualitativen Dignität «FA Interventionelle Schmerztherapie» versehen und können nur durch Ärzte, die über den FA Interventionelle Schmerztherapie verfügen, erbracht und dem Kostenträger in Rechnung gestellt werden. Zudem sind alle Tarifpositionen dieses Unterkapitels, mit Ausnahme der Tarifposition 29.2000 Behandlung und problemorientierte Untersuchung durch den Facharzt mit FA Interventionelle Schmerztherapie, pro 5 Minuten mit der Sparte «Interventionelle Schmerztherapie» tarifiert. Diese Sparte wird gemäss demnächst aktualisiertem Spartenkonzept eine Anerkennungssparte, welche auf Antrag durch die paritätische Kommission Dignitäten und Sparten PaKoDig offiziell anzuerkennen ist. Zur Erlangung der Spartenanerkennung ist der FA «Interventionelle Schmerztherapie» die Voraussetzung. Inhaber von Besitzstandspositionen können den FA «Interventionelle Schmerztherapie» bei der SSIPM bis ein Jahr nach Inkraft treten der Version 1.08 beantragen.

\section{Unterkapitel 00.03.05 Komplementär- medizin - KLV-Änderung vom 31. Mai 2011}

Aufgrund der KLV-Änderung vom 31. Mai 2011 stellen neu - neben der Akupunktur (00.1710/20/ 30/35) - auch die folgenden komplementärmedizinischen Leistungen im Zeitraum vom 01.01.2012 bis 31.12.2017 (Evaluationsphase) eine Pflichtleistung dar:

- Anthroposophische Medizin (00.1840/50/60),

- Arzneimitteltherapie der Traditionellen Chinesischen Medizin (TCM) (00.1810/20/30),

- Ärztliche Klassische Homöopathie(00.1770/ 80/90 und 00.1800),

- Phytotherapie (00.1870 bzw. 00.0010/20/30),

\section{Tabelle 1}

Leistungen, die neu in das Tarifwerk TARMED aufgenommen wurden.

\begin{tabular}{|c|c|c|c|}
\hline Position & Leistung & Qualitative Dignität & Bemerkung \\
\hline 04.0490 & $\begin{array}{l}\text { Extrakorporelle } \\
\text { Photophorese }\end{array}$ & $\begin{array}{l}\text { Dermatologie und Venerologie, Hämato- } \\
\text { logie, Onkologie-Hämatologie, SP pädiatri- } \\
\text { sche Onkologie-Hämatologie }\end{array}$ & $\begin{array}{l}\text { Pflichtleistung unter bestimmten Voraussetzun- } \\
\text { gen (siehe KLV) }\end{array}$ \\
\hline 09.1785 & $\begin{array}{l}\text { Implantation einer Knochenverankerung für } \\
\text { das Hörgerät BAHA }\end{array}$ & ORL, speziell Hals-/ Gesichtschirurgie & $\begin{array}{l}\text { Pflichtleistung unter bestimmten Voraussetzun- } \\
\text { gen (siehe KLV) }\end{array}$ \\
\hline 19.0170 & $\begin{array}{l}\text { Atemtest mit Harnstoff } 13 \mathrm{C} \text { zum Nachweis von } \\
\text { Helicobacter pylori durch nichtärztliches Personal }\end{array}$ & - & Beinhaltet die vollständige Abwicklung des Tests \\
\hline 21.2125 & $\begin{array}{l}\text { Implantation von Goldmarkern zur Bestrahlungs- } \\
\text { planung der Prostata }\end{array}$ & Urologie & $\begin{array}{l}\text { Pflichtleistung unter bestimmten Voraussetzun- } \\
\text { gen (siehe KLV) }\end{array}$ \\
\hline 32.0685 & $\begin{array}{l}\text { MRI bei Stereotaktischer Radiochirurgie - Einmal- } \\
\text { bestrahlung mit Gamma-Knife-Technik }\end{array}$ & $\begin{array}{l}\text { Angiologie, Medizinische Radiologie/ } \\
\text { Radiodiagnostik und Neuroradiologie }\end{array}$ & - \\
\hline 32.0695 & $\begin{array}{l}\text { CT bei Stereotaktischer Radiochirurgie - } \\
\text { Einmalbestrahlung mit Gamma-Knife-Technik }\end{array}$ & $\begin{array}{l}\text { Angiologie, Medizinische Radiologie/ } \\
\text { Radiodiagnostik und Neuroradiologie }\end{array}$ & - \\
\hline
\end{tabular}


- Störfeldtherapie (Neuraltherapie nach Hunke) (00.1740/50/60) und

- telefonische komplementärmedizinische Konsultation durch den Facharzt (00.1880/90/ 00.1900).

\section{Leistungen, die neu in das Tarifwerk TARMED aufgenommen wurden}

Eine Übersicht findet sich in Tabelle 1 auf Seite 12.

\section{Besuchs-Inkonvenienzpauschale}

\section{Mo.-Fr. 07-19.00 Uhr, Sa. 07.00-12.00 Uhr}

Tarifposition 00.0065 Besuchs-Inkonvenienzpauschale Mo.-Fr. 07-19.00 Uhr, Sa. 07.00-12.00 Uhr: Mit Schreiben vom 21.4.2011 teilte das Bundesamt für Gesundheit BAG der Geschäftsstelle TARMEDSuisse mit, dass der Bundesrat an der Sitzung vom 20. 4.2011 entschieden hat, die Besuchs-Inkonvenienzpauschale (Tarifposition 00.0065 Besuchs-Inkonvenienzpau-

Tabelle 2

Tarifpositionen, bei denen die qualitative Dignität ergänzt wurde.

\begin{tabular}{|c|c|c|c|}
\hline Position & Leistung & Qualitative Dignität ergänzt mit: & Qualitative Dignitäten neu \\
\hline 03.0310 & $\begin{array}{l}\text { Entwicklungspädiatrische Untersuchungen beim Kind } \\
\text { bis } 7 \text { Jahre, partiell, pro } 5 \text { Min. }\end{array}$ & SP Entwicklungspädiatrie & $\begin{array}{l}\text { Neonatologie } \\
\text { SP Entwicklungspädiatrie } \\
\text { SP Neuropädiatrie }\end{array}$ \\
\hline 03.0320 & $\begin{array}{l}\text { Entwicklungspädiatrische Untersuchungen beim Kind } \\
\text { bis } 7 \text { Jahre, umfassend, pro } 5 \text { Min. }\end{array}$ & SP Entwicklungspädiatrie & $\begin{array}{l}\text { SP Entwicklungspädiatrie } \\
\text { SP Neuropädiatrie }\end{array}$ \\
\hline 03.0330 & $\begin{array}{l}\text { Entwicklungspädiatrische Untersuchungen beim Kind/ } \\
\text { Jugendlichen ab } 7 \text { bis } 18 \text { Jahre, partiell, pro } 5 \text { Min. }\end{array}$ & SP Entwicklungspädiatrie & $\begin{array}{l}\text { SP Entwicklungspädiatrie } \\
\text { SP Neuropädiatrie }\end{array}$ \\
\hline 03.0340 & $\begin{array}{l}\text { Entwicklungspädiatrische Untersuchungen beim Kind/ } \\
\text { Jugendlichen ab } 7 \text { bis } 18 \text { Jahre, umfassend, pro } 5 \text { Min. }\end{array}$ & SP Entwicklungspädiatrie & $\begin{array}{l}\text { SP Entwicklungspädiatrie } \\
\text { SP Neuropädiatrie }\end{array}$ \\
\hline 17.0410 & $\begin{array}{l}\text { Arterieller Gefässstatus mittels CW-Doppler, obere und } \\
\text { untere Extremitäten }\end{array}$ & FA Phlebologie & $\begin{array}{l}\text { Angiologie } \\
\text { FA Phlebologie }\end{array}$ \\
\hline 17.0490 & $\begin{array}{l}\text { Bidirektionale Doppleruntersuchung der oberflächlichen } \\
\text { und/oder tiefen Venen einer oberen/unteren Extremität, } \\
\text { pro Seite }\end{array}$ & FA Phlebologie & $\begin{array}{l}\text { Angiologie } \\
\text { FA Phlebologie }\end{array}$ \\
\hline 39.3510 & $\begin{array}{l}\text { Gefäss-Sonografie im Rahmen einer } \\
\text { Organ- oder Weichteiluntersuchung }\end{array}$ & $\begin{array}{l}\text { Gynäkologie und Geburtshilfe, Gastro- } \\
\text { enterologie, Neurologie, Urologie, } \\
\text { FA Ultraschall Schwangerschaft } \\
\text { und FA Zerebrovaskuläre Krankheit }\end{array}$ & $\begin{array}{l}\text { Angiologie } \\
\text { FA Sonografie Modul Bewegungsapparat } \\
\text { FA Sonografie Modul Gynäkologie } \\
\text { FA Sonografie Modul Abdomen } \\
\text { FA Sonografie Modul Gefässe } \\
\text { FA Sonografie Modul Halsorgane } \\
\text { FA Sonografie Modul Mammasonografie } \\
\text { FA Sonografie Modul Pädiatrie } \\
\text { FA Ultraschall Schwangerschaft } \\
\text { FA Zerebrovaskuläre Krankheit } \\
\text { Gastroenterologie } \\
\text { Gynäkologie und Geburtshilfe } \\
\text { Medizinische Radiologie/Radiodiagnostik } \\
\text { Neurologie }\end{array}$ \\
\hline 39.3520 & $\begin{array}{l}\text { Applikation von Kontrastmittel } \\
\text { oder Pharmakostimulation }\end{array}$ & $\begin{array}{l}\text { Gynäkologie und Geburtshilfe, } \\
\text { Gastroenterologie, Urologie, } \\
\text { FA Zerebrovaskuläre Krankheit }\end{array}$ & $\begin{array}{l}\text { Angiologie } \\
\text { FA Sonografie Modul Bewegungsapparat } \\
\text { FA Sonografie Modul Gynäkologie } \\
\text { FA Sonografie Modul Abdomen } \\
\text { FA Sonografie Modul Gefässe } \\
\text { FA Sonografie Modul Halsorgane } \\
\text { FA Sonografie Modul Mammasonografie } \\
\text { FA Sonografie Modul Pädiatrie } \\
\text { FA Zerebrovaskuläre Krankheit } \\
\text { Gastroenterologie } \\
\text { Gynäkologie und Geburtshilfe } \\
\text { Medizinische Radiologie/Radiodiagnostik } \\
\text { Urologie }\end{array}$ \\
\hline 39.3600 & Gefäss-Sonografie viszerale Arterien & $\begin{array}{l}\text { Gynäkologie und Geburtshilfe, } \\
\text { Gastroenterologie und Urologie }\end{array}$ & $\begin{array}{l}\text { Angiologie } \\
\text { FA Sonografie Modul Gynäkologie } \\
\text { FA Sonografie Modul Abdomen } \\
\text { FA Sonografie Modul Gefässe } \\
\text { FA Sonografie Modul Pädiatrie } \\
\text { Gastroenterologie } \\
\text { Gynäkologie und Geburtshilfe } \\
\text { Medizinische Radiologie/Radiodiagnostik } \\
\text { Urologie }\end{array}$ \\
\hline
\end{tabular}


schale) vom 1.1.2011 bis 31.3.2012 zu genehmigen bzw. zu verlängern. Der Leitungsgremium-Ausschuss LGA hat dann aufgrund von Divergenzen mit der Tarifpartnerin santésuisse, die eine Weiterführung der BIP aus unerfindlichen Gründen verweigert, an der Sitzung vom 11.7.2011 Folgendes beschlossen:

- Die Tarifposition 00.0065 + Besuchs-Inkonvenienzpauschale Mo.-Fr. 07.00-19.00 Uhr, Sa. 07.00-12.00 Uhr kann aus oben erwähnten Gründen nicht mehr in der Tarifstruktur TARMED Version 1.08, die per 1.6.2012 in Kraft tritt, verankert sein und

- die Weiterführung der Tarifposition 00.0065 + Besuchs-Inkonvenienzpauschale Mo.-Fr. 07.00-19.00 Uhr, Sa. 07.00-12.00 Uhr ausserhalb der Tarifstruktur TARMED wird zwischen den die Besuchs-Inkonvenienzpauschale befürwortenden Parteien (FMH und diversen Krankenkassen) in separaten Vereinbarungen ausserhalb dem Tarifwerk TARMED geregelt.

\section{Tarifpositionen, die neu mit der Weg- entschädigung kumuliert werden können}

Folgende Tarifpositionen können neu mit der Tarifposition 00.0095 + Wegentschädigung, pro 5 Minuten in einer Sitzung kumuliert werden, wenn die Leistung nicht in der Arztpraxis, sondern vor Ort beim Patienten erbracht wird:

- 00.2120 Erweiterte konsiliarische Beratung (Konsilium) durch den Facharzt, pro 5 Minuten

- 02.0010 Psychiatrische Diagnostik und Therapie, Einzeltherapie, erste Sitzung, pro 5 Minuten

- 02.0020 Psychiatrische Diagnostik und Therapie, Einzeltherapie, jede weitere Sitzung, pro 5 Minuten

\section{Tarifpositionen, bei denen die qualitative Dignität ergänzt wurde}

Eine Übersicht findet sich in Tabelle 2 .

\section{Diverse sonstige Änderungen bestehender Tarifpositionen}

Dermatologische Lasertherapie durch den Facharzt: Die dermatologische Lasertherapie ist neu anhand der folgenden drei anstatt 9 Tarifpositionen tarifiert: 04.0370 Dermatologische Lasertherapie durch den Facharzt jede Laser-Methode, erste 5 Minuten; 04.0380 Dermatologische Lasertherapie durch den Facharzt jede Laser-Methode, jede weiteren 5 Min. und 04.0390 Dermatologische Lasertherapie durch den Facharzt jede Laser-Methode, letzte 5 Minuten. Zudem wurde die dermatologische Lasertherapie mit der qualitativen Dignität Dermatologie und Venerologie ergänzt. Es gilt zu beachten, dass die dermatologische Lasertherapie eine Pflichtleistung unter bestimmten Voraussetzungen (siehe Indikation gemäss KLV) ist.
Grundkonsultation/Betriebsstelle Radiologie im Röntgeninstitut ausserhalb Spital/ im Spital KVG und UVG/ $M V G / I V G$ : Neu gilt für die folgenden Tarifpositionen die Regel Menge «Pro Sparte 1-mal pro Tag; jedoch max. 2-mal pro Tag»: 39.0010 Grundkonsultation/Betriebsstelle Radiologie im Röntgeninstitut ausserhalb Spital, KVG, 39.0011 Grundkonsultation/Betriebsstelle Radiologie im Röntgeninstitut ausserhalb Spital, UVG/MVG/IVG sowie 39.0015 Grundkonsultation/Betriebsstelle Radiologie im Spital, KVG, 39.0016 Grundkonsultation/Betriebsstelle Radiologie im Spital, UVG/MVG/IVG.

\section{Analyseliste - befristete Weiterführung des Übergangszuschlags für das Praxislabor} Der Übergangszuschlag (Tarifposition 4708.00) der Analyseliste wird für das Praxislabor gemäss Entscheid des Eidgenössischen Departements des Innern (Krankenpflege-Leistungsverordnung, KLV, Anhang 3 - Änderung per 1.1.2012) um ein Jahr befristet auf den 31.12.2012 verlängert. Damit wurde dem Antrag der FMH entsprochen.

Auf den 1.1.2012 treten zudem bei der Vergütung von medizinischen Leistungen, Laboranalysen sowie der Liste der Mittel und Gegenstände MiGeL verschiedene Änderungen in Kraft. Das Eidgenössische Departement des Innern (EDI) hat die KrankenpflegeLeistungsverordnung KLV und ihre Anhänge entsprechend angepasst. Die weiteren Änderungen bei Krankenkassenvergütungen sind unter www.news.admin.ch/message/index.html?lang=de\&msg-id=42798 einsehbar.

\section{Schlussbemerkung}

Die hier aufgelisteten Änderungen stellen eine selektive und nicht abschliessende Auflistung wichtiger Änderungen, die mit der Tarifversion 1.08 per 1.6.2012 eingeführt werden, dar. Eine abschliessende Liste aller Änderungen (inkl. rein technischer Änderungen, textlicher Änderungen usw.) können dem Änderungsdokument, das unter dem folgenden Link aufgeschaltet ist, entnommen werden: www.tarmedsuisse.ch / Änderungsprotokoll

\section{Danke und alles Gute für 2012}

Das ganze Team vom Ressort Tarife und Verträge der FMH und die beiden für das Ressort verantwortlichen Zentralvorstandsmitglieder Dr. med. Ernst Gähler und Dr. med. Remo Osterwalder möchten sich bei Ihnen ganz herzlich für Ihre Unterstützung und Ihre konstruktive Mitarbeit im vergangenen Jahr bedanken.

Wir freuen uns auf ein neues, sicher wieder interessantes Jahr und auf die weitere gute Zusammenarbeit. Ihnen und Ihren Familienangehörigen wünschen wir alles Gute, gute Gesundheit und viel Schönes für das Jahr 2012. 